\title{
Next generation mouse models of squamous cell lung cancer for translational immuno-oncology
}

\section{Josephine Hai}

With immunotherapy rapidly becoming a mainstay of cancer treatment, a more comprehensive understanding of the immune microenvironment is essential. Patientderived xenografts (PDX) lack the endogenous immune microenvironment, and humanized mice, though promising, often do not accurately recapitulate basic immunological features, such as correct abundance of major leukocytic lineages. Genetically engineered mouse models (GEMMs) and transplantable tumors to syngeneic immunocompetent mice are often necessary to test novel immunotherapies and identify potential predictive biomarkers.

Historically, the development of therapies for lung squamous cell carcinoma (LSCC) has significantly lagged, due in part to the lack of animal models with pure squamous histology and patient-relevant mutant genotypes. The development of LSCC GEMMs has been met with many challenges. For one, in vivo interrogation of the complex mutational landscape of LSCC, which was only revealed in 2012 from The Cancer Genome Atlas (TCGA), has been limited by the resource-intensive need to generate and crossbreed mice bearing multiple, distinct engineered inducible mutant alleles [1]. Unlike lung adenocarcinoma, no single driver oncogene has been validated for LSCC. Furthermore, these GEMMs often require tissue-specific cre-lox systems, which pose challenges for squamous cell carcinomas since this can lead to premature termination of mice that develop skin lesions before the onset of LSCCs [2]. Xu et al. previously demonstrated that bi-allelic inactivation of Pten and Stk11 in mice gave rise to LSCCs with high PD-L1 expression after 40 to 50 weeks [3]. Ferone et al. have also developed LSCC transgenic mice harboring Sox 2 overexpression with bi-allelic inactivation of Pten and Cdkn2ab after 7-9 months with a 73\% penetrance [4]. The long latency periods for the development of LSCC in GEMMs (7-9 months) significantly hampers preclinical testing of immunotherapies. Additionally, cell lines derived from the primary murine tumors do not always grow in culture to enable transplantable syngeneic tumor models, presumably due in part to p53-activation dependent growth arrest, and require subsequent steps of artificial immortalization with SV40 T-antigen [5].

To overcome these barriers, we and others have efficiently engineered genetically-defined immunocompetent mouse models by leveraging both organoid and CRISPR-based methodology [6-8]. We engineered lung organoids derived from Cre-dependent Sox 2 knock-in mice bearing three tumor suppressor gene mutations co-occurring in human LSCC (Tp53 $3^{\text {indel } / P t e n} n^{\text {indel/ }}$ $\left.C d k n 2 a^{\text {indel }}\right)$ and capable of forming tumors in syngeneic immunocompetent hosts with significantly shorter latencies than traditional crossbreeding strategies [6]. These transformed organoids give rise to SCC that closely mirror human tumor histology, signaling alterations, and immune-stromal-tumor microenvironment [6]. Using this new immunocompetent mouse model, we demonstrated efficacy of combining immune checkpoint inhibitors with DNA-damage inducing targeted therapy for this genotype of LSCC [6].

Rapid generation of a panel of immunocompetent mouse models of complex genotypes using this approach would be a powerful platform for characterizing genotypespecific sensitivities to immunotherapies for LSCC and could be adapted to high throughput drug screens. Studies from other cancer types have begun to bring this concept to fruition. O'Rourke et al. previously highlighted that use of ex vivo engineered mouse organoids derived from GEMMs provided a flexible, fast, and low-cost platform to model all stages of colorectal cancer that recapitulated the entire adenoma-adenocarcinoma-metastasis axis in vivo [8]. More recently, Zhang et al. generated multiple high grade serous tubo-ovarian carcinoma (HGSC) models exhibiting a panel of genotypes frequently observed in patients by CRISPR engineering fallopian tube epithelial (FTE) organoids derived from Tp53fff GEMMs [7]. Using these syngeneic organoid models, they demonstrated that tumors with $T p 53^{--} / C \mathrm{Cne} 1^{O E} / A k t 2^{O E} / \mathrm{Kras}^{O E}$ genotype were highly sensitive to chemotherapy and immunotherapy combinations (gemcitabine plus anti-PD-L1 and antiCTLA-4), while Tp53-- $/$ Pten $^{-/} / N f 1^{-/}$tumors were not [7]. Similarly, the Weinberg group also demonstrated the utility of engineering ex vivo murine FTE cells bearing complex mutational combinations (up to five co-occurring genetic alterations) and forming a panel of five genotypespecific models that grew in syngeneic hosts [9]. By treating these new mouse models with a combination of immune checkpoint inhibitors and chemotherapies, they identified follistatin as a driver of resistance to immune checkpoint inhibitors in Ccnel-overexpressing $\left(\mathrm{Ccnel}^{\mathrm{OE}}\right)$ mice [9].

Leveraging emerging technologies in both CRISPR/ Cas 9 mutagenesis and organoid culturing methods offers hope that enhanced development of unique clinically 
relevant mouse models will shed light on the complex tumor-immune microenvironment, enabling more efficient preclinical testing to understand how different patient subsets may respond to novel immunotherapies. This powerful approach holds promise to predict genotypedriven drug responses in a personalized fashion, especially for LSCC patients, who have had very limited therapeutic options for many years.

\section{CONFLICTS OF INTEREST}

Authors have no conflicts of interest to declare.

Josephine Hai: Department of Medical Oncology, DanaFarber Cancer Institute, Boston, MA 02215, USA

Correspondence to: Josephine Hai, email josephine.hai@utoronto.ca

Keywords: squamous cell lung cancer; mouse models; immunotherapy; CRISPR

Received: November 15, 2020

Published: December 01, 2020

\section{REFERENCES}

1. Cancer Genome Atlas Research Network. Nature. 2012; 489:519-25. https://doi.org/10.1038/nature11404. [PubMed]
2. Kirkley KS, et al. Comp Med. 2017; 67:407-15. [PubMed]

3. Xu C, et al. Cancer Cell. 2014; 25:590-604. https://doi. org/10.1016/j.ccr.2014.03.033. [PubMed]

4. Ferone G, et al. Cancer Cell. 2016; 30:519-32. https://doi. org/10.1016/j.ccell.2016.09.001. [PubMed]

5. Eichner LJ, et al. bioRxiv. 2020. https://doi. org/10.1101/2020.10.14.338590.

6. Hai J, et al. Clin Cancer Res. 2020; 26:3431-42. https://doi. org/10.1158/1078-0432.Ccr-19-1627. [PubMed]

7. Zhang S, et al. Cancer Discov. 2020. https://doi. org/10.1158/2159-8290.Cd-20-0455. [PubMed]

8. O'Rourke KP, et al. Nat Biotechnol. 2017; 35:577-82. https://doi.org/10.1038/nbt.3837. [PubMed]

9. Iyer S, et al. Cancer Discov. 2020. https://doi. org/10.1158/2159-8290.Cd-20-0818. [PubMed]

Copyright: (c) 2020 Hai. This is an open access article distributed under the terms of the Creative Commons Attribution License (CC BY 3.0), which permits unrestricted use, distribution, and reproduction in any medium, provided the original author and source are credited. 